Portland State University

PDXScholar

1982

\title{
A study of factors affecting cognitive style in Mexican-American children
}

Anne Kelter Gehrig

Portland State University

Follow this and additional works at: https://pdxscholar.library.pdx.edu/open_access_etds

Part of the Cognitive Psychology Commons, and the Education Commons Let us know how access to this document benefits you.

\section{Recommended Citation}

Gehrig, Anne Kelter, "A study of factors affecting cognitive style in Mexican-American children" (1982). Dissertations and Theses. Paper 3182.

https://doi.org/10.15760/etd.3171

This Thesis is brought to you for free and open access. It has been accepted for inclusion in Dissertations and Theses by an authorized administrator of PDXScholar. Please contact us if we can make this document more accessible: pdxscholar@pdx.edu. 
AN ABSTRACT OF THE THESIS OF Anne Kelter Gehrig for the Master of Arts in Education presented February 5, 1982.

Title: A Study of Factors Affecting Cognitive Style in Mexican-American Children.

APPROVED BY MEMBERS OF THE THESIS COMMITTEE:
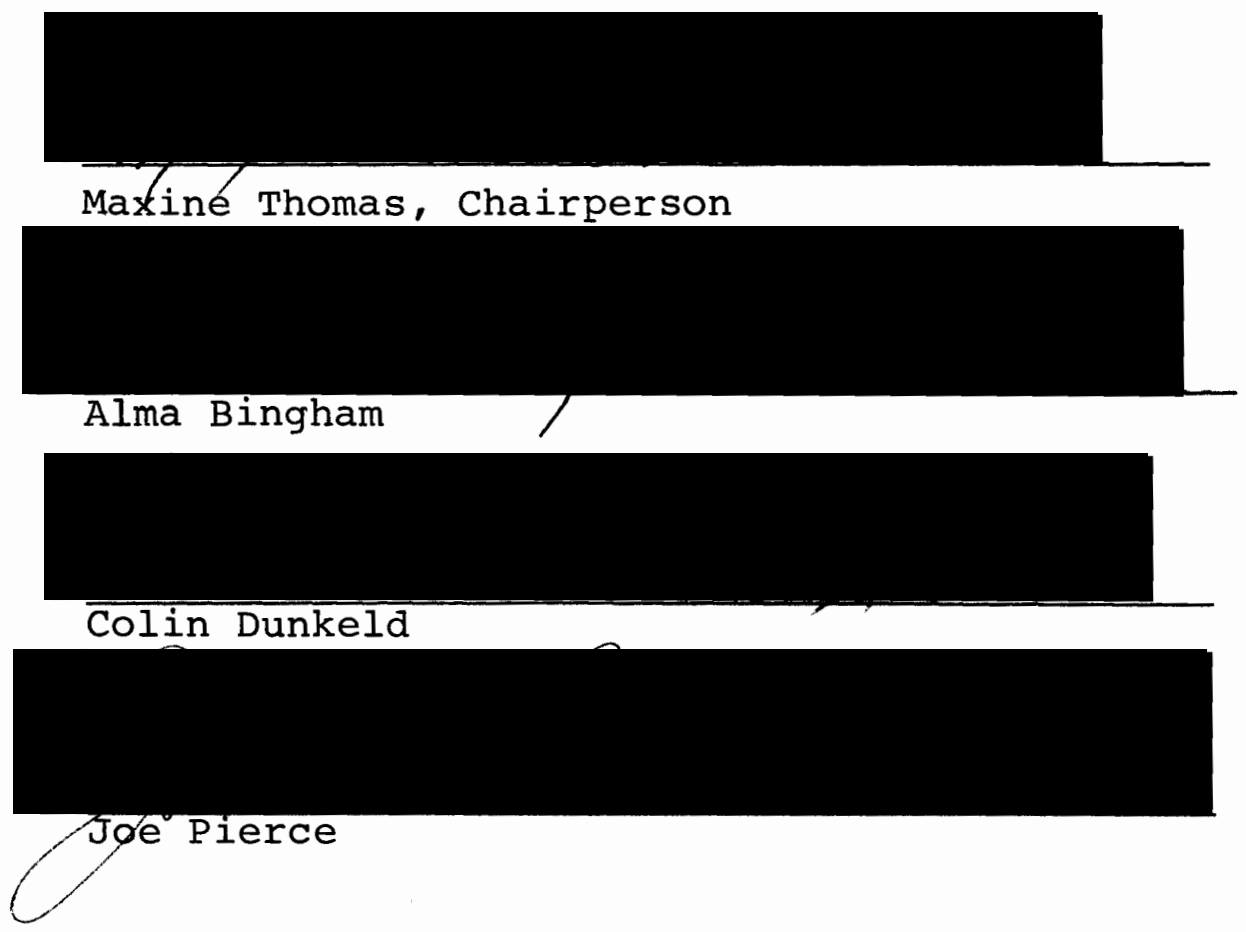

In recent years cognitive style has become the focus of much educational research. Many educators believe that instructional effectiveness can be maximized by utilizing the student's preferred cognitive style as a springboard for introducing new concepts as well as by restructuring educational practices so that the child learns to deal effectively with diverse cognitive styles. Early research on cognitive style focused solely on behavioral indicators. Today many research- 
ers acknowledge that, in order to understand cognitive style, one must examine brain processing, as different styles of thinking result from the manner in which each hemisphere of the brain processes information.

This study, based on the investigator's observations of reading behaviors of Mexican-American and Anglo-American children, and on an examination of available research on cognitive style and hemispheric functioning, has a threefold purpose:

1) to identify learning styles as observed in the classroom,

2) to define cognitive style in terms of neuropsychological research, and

3) to identify major factors affecting cognitive style. Major factors investigated include language usage, language structure, family organization, and socioeconomic variables. Of these, language usage appears to be the most significant factor; it, in turn, is influenced by socioeconomic level and family organizational practices.

Cognitive styles depend upon stimulation for their proper development; social variables may determine the amount and quality of stimulation afforded a particular hemisphere. The data gathered is examined in light of its implications for educational practices, and recommendations are made for utilizing the results of cognitive style research in the classroom situation. 


\title{
A STUDY OF FACTORS AFFECTING COGNITIVE STYLE IN MEXICAN-AMERICAN CHIIDREN
}

$$
\text { by }
$$

ANNE KELTER GEHRIG
A thesis submitted in partial fulfillment of the requirements for the degree of

\author{
MASTER OF ARTS \\ in \\ EDUCATION
}

Portland State University

1982 
TO THE OFFICE OF GRADUATE STUDIES AND RESEARCH:

The members of the Committee approve the thesis of Anne Kelter Gehrig presented February 5, 1982.

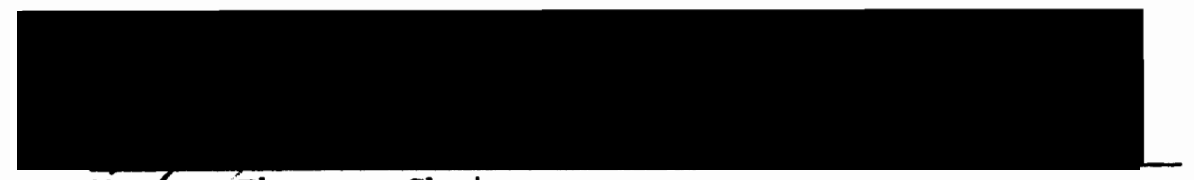

Maxine Thomas, Chairperson
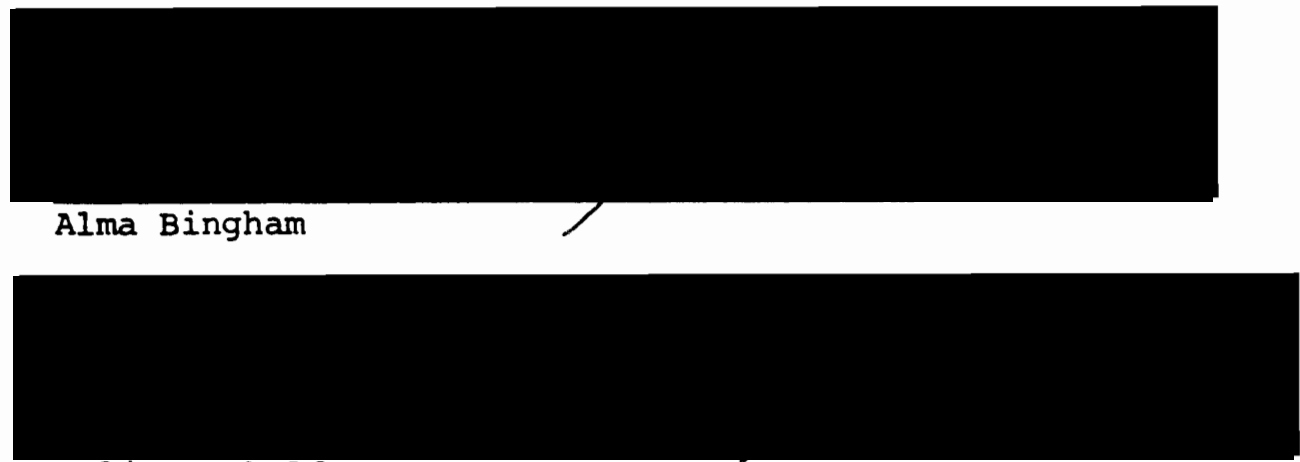

Colin Dunkeld

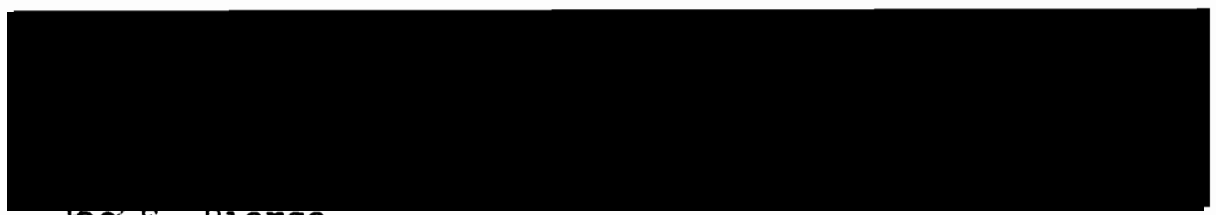

Jog E. Plerce

APPROVED :

Donald Leu, Dean of Education

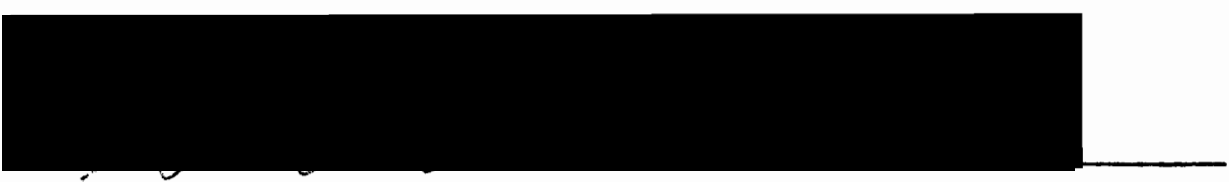

Stanley Rauch, - Dean of Graduate Studies 


\section{ACKNOWLEDGEMENTS}

This writer would like to express appreciation to the members of her thesis committee for their suggestions and constructive criticism. She is especially indebted to Dr. Maxine Thomas for her invaluable help and support.

Appreciation is also extended to James Carnes for the books and articles he shared, and to the members of the writer's family for their help, encouragement, and support. 
TABLE OF CONTENTS

PAGE

ACKNOWLEDGEMENTS . . . . . . . . . . . . . . . . . . . . . . . iii

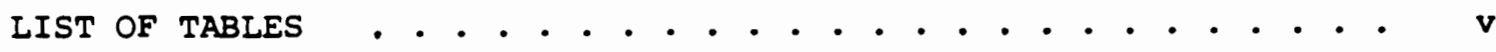

CHAPTER

I INTRODUCTION. . . . . . . . . . . . . . . . . 1

II DEFINITION OF TERMS . . . . . . . . . . . . . . . 4

Cognitive style . . . . . . . . . . . . . 4

Hemispheric Function and Cognitive Style... . . . 5

II EFFECTS OF LANGUAGE ON COGNITIVE STYLE. . . . . . . . . 13

Structure . . . . . . . . . . . . . . 13

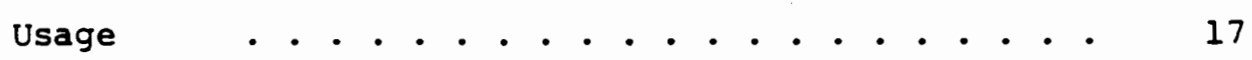

IV RELATIONSHIP BETWEEN SOCIALIZATION PRACTICES

AND IINGUISTIC CODE . . . . . . . . . . . . . . 21

V ACCULTURATION, LINGUISTIC CODE, AND COGNITIVE STYLE • . 28

Socioeconomic Level .. . . . . . . . . 30

Bilingualism . . . . . . . . . . . . . . 32

Education . . . . . . . . . . . . . 33

VI CONCLUSIONS AND RECOMMENDATIONS . . . . . . . . . . . 35

Implications . . . . . . . . . . . . 38

Application . . . . . . . . . . . . . 39

BIBLIOGRAPHY . . . . . . . . . . . . . . . . . . . . . . 4 41 


\section{LIST OF TABLES}

I Characteristics of Left and Right Hemispheric Functioning

II Features of Mexican-American and

Anglo-American Value Systems . . . . . . . . . . 26 


\section{CHAPTER I}

\section{INTRODUCTION}

This thesis is the result of information gleaned from three years of graduate work and research in elementary education, and draws heavily from five years of personal experience in the field. It began with observations of children engaged in the reading process and ended with research into the cognitive processes involved in reading.

Initial experience as a Title 1-M Teacher/Home-School Consultant entailed working as a resource person with migrant children, both in the school and in the home. Some were Anglo, but most were Mexican or Mexican-American. Some made the migrant circuit from California to Washington on a regular basis; others stayed while their families sought more steady work in the packing houses and light manufacturing plants. Frequent moves had disrupted the education of many of the children and a limited fluency in English often interfered with learning. As a resu't a large number of these children were academically behind their Feers and unable to function in the regular classroom without additional help. Teaching in the Title $1-M$ program involved teaching English as a second language and providing supplementary instruction in reading, math, and other basis subject areas.

Working in small groups and on a one-to-one basis with these migrant children provided ample opportunity for informal observation of reading behaviors. Each child had his or her own mechanisms for coping with reading roadblocks. Maria, when stymied by a word, thumbed through 
the book until she found where she had previously encountered it. She then returned to her original place and resumed reading. Hilda used a similar approach, except that she looked to bulletin boards and experience charts for help. Flora would read a word and repeat it in Spanish, holding up her hands to indicate the relative length of both words. Dunia would stand near the reading table for long periods of time, holding a copy of a primer and intoning, "This one looks like this one. They're the same," as she pointed to words and phrases.

Perhaps the most intriguing approach was that of Pablo who, despite three years of intensive instruction in phonics and direct instructional programs, still had not even a rudimentary knowledge of phoneme-grapheme correspondences. He was quite literally a non-reader. When an attempt was made to teach him whole words in context, he slowly began to read. His miscues were consistent, syntactically correct, yet often humorous. "Funny John" replaced "Silly Sam" in one story, and "the horse leaped over the creek" became "the pony jumped over the lake" in another. Interwoven through all of these examples was a common thread: a reliance on the visual and contextual aspects of a word. These experiences in the migrant program contrasted sharply with those in the regular classroom. There seemed to be a marked contrast among the Mexican and Mexican-American and Anglo-American students. While the migrant children were attending to the visual similarities of words, phrases, and sentences and making syntactically-acceptable substitutions, the non-migrant Anglo children were teaching themselves to read by "sounding it out". Upon encountering the word "hill", for example, the former might substitute "mountain", while the latter might be be more likely to respond, "H-i-l-l, hill:" Phonics programs and direct 
instruction were much more successful with the Anglo students than with their non-Anglo classmates. To use the textbook terminology, it seemed that the former were learning "analytically', piecing together words and phrases from component sounds, while the latter preferred a "synthetic" approach, beginning with whole words and phrases breaking them down.

The purpose of this study is to explore the cognitive styles of reading behaviors of Mexican-American and Anglo-American children. To fulfill this purpose, the study will:

1) identify learning styles as observed by the investigator,

2) define cognitive style in terms of recent neuropsychological research, and

3) identify major factors which affect cognitive style.

Chapter II will define cognitive style in terms of recent neuropsychological research and will describe differences in cognitive processing within the two subcultures. Chapters III, IV, and V will examine some of the major factors which are thought to have an effect on cognitive style. In Chapter VI the applications to reading instruction will be examined. 
CHAPTER II

DEFINITION OF TERMS

COGNITIVE STYLE

Cognitive style is not easy to define, since it deals with the mind. Because it is impossible to completely share another's experience --to literally "read one's mind"--one must rely upon observation of behavorial indicators. Perhaps for that reason much of the literature on cognitive style is descriptive rather than definitive in nature. Cognitive styles are contrasted, labelled, and described in detail, but attempts to define the nature of cognitive style are vague and elusive. Witkin (1967) states that cognitive styles refer to differences in how one perceives; he then proceeds to describe those differences. For example, field-dependent individuals have a superior memory for social information such as facial cues, while field-independent persons respond to stimuli independent of its environment, e.g. focusing on significant details as separate from the overall "big picture". Similarly, Wittrock (1979) describes cognitive style as "stable ways people differ in perception, encoding, and storage of information."

Much of the earlier cognitive research focused on the perceptual dimension known as 'field-dependence/field-independence'. Although the term originally referred to an individual's spatial orientation, it became apparent that a person's perceptual style was indicative of his thinking or cognitive style (Dixon 1977). 
Greater differentiation shows itself in the intellectual domain... in a tendency to experience items as separate from context...with this extension to the picture of self-consistency from perceptual to intellectual domains, the label of 'cognitive styles' becomes appropriate as a more comprehensive concept than 'perceptual styles' (Witkin, Berry 1975).

Gradually the list of dichotomies used to describe that area of intellectual functioning grew: analytic/synthetic (Brooks 1978, Buriel 1978, Wittrock 1977), global/articulated (Witkin 1967), serialistic/ holistic (Entwhistle 1979) are a few of the more frequently-used labels. Labels differ, but the underlying concept is the same: descriptions of the ways in which an individual deals with information. Labels and descriptions are not definitions however. They tell what a certain cognitive style is like, but not what cognitive style itself actually is.

HEMISPHERIC FUNCTION AND COGNITIVE STYLE

In the past decade, great strides have been made in the field of neurology, yielding a better understanding of the way in which the brain functions. By observing the brain, it is possible to obtain information about the mind that would be otherwise inaccessible were one to rely, as in the past, upon language and behavior (Ornstein 1972). The brain has become the passport to the mind.

As early as the nineteenth century it has been known that the brain consists of two similar yet asymmetrically-functioning hemispheres (Cohen 1977. Gazzaniga, LeDoux 1978). Early information about their functioning was derived from the study of brain-damaged individuals. Performance of patients with localized damage to one hemisphere was compared with that of persons sustaining similar damage in the opposite hemisphere. The kinds of deficits incurred varied according to which hemisphere sustained the 
damage. Right hemisphere lesions result in deficits of a different nature than those incurred by left hemisphere lesions (Cohen 1977). There are many limitations to this sort of research. Secondary complications may occur as a result of an injury, thus making it difficult to clearly establish a causal relationship between the lesion and the resulting deficit. Because cases of left hemisphere injury must be closely matched with those of right hemisphere injury, and because additional factors such as age, I.Q., handedness, and sex must also be taken into consideration, the number of cases available for study is quite limited. The undamaged area is often able to partially compensate for deficits in the opposite hemisphere, making it difficult to assess the degree of overlap, and the percentage of individuals who differ as a result. Nevertheless, it has been established that, in most cases, right hemisphere lesions result in deficits in visual-spatial functioning and left hemisphere lesions are associated with deficits in the ability to perform verbal tasks (Cohen 1977).

Today evidence of hemispheric function is derived from two additional sources: work with split-brain patients, and work with normalbrain subjects. While it is again possible to take issue with the methodology and interpretation of the findings, the comparison of results in the three types of studies yields information which converges to such a great extent that "the general conclusions that different cognitive processes are subserved by different hemispheres are not in doubt" (Cohen 1977).

Results of work by Sperry and Gazzaniga (Cohen 1977, Springer and Deutsch 1981) with split-brain subjects reinforce conclusions made as a result of research with brain-damaged patients. At the same time, 
split-brain research avoids the problem of take-over functioning by the undamaged hemisphere. The corpus callosum of epileptic patients was surgically severed with the intent of reducing the severity of seizures by interrupting the communication network between the two hemispheres. By severing the tissue connecting the hemispheres, it was possible to assess the capabilities of each side in isolation because information could no longer be transferred from one side to the other.

Since the left hemisphere controls functioning of the right visual field and the right hemisphere controls functioning of the left visual field, researchers have devised experiments in which information is presented to one hemisphere via the contralateral visual field. In one such experiment, faces composed of two unmatching halves were shown to split-brain patients. The left half, for example might be a boy's face while the right might be that of a woman. The "chimeric stimuli" was presented separately to each hemisphere. When the patient was asked to give a verbal description of what he saw, he reported the face in the right visual field. When asked to give a visual gestalt response (pointing) to the same question, he indicated the face in the left visual field (Springer and Deutsch 1981, Gazzaniga and LeDoux 1978). By channeling stimuli to one or the other hemisphere through the contralateral visual field and observing the patient's response, sperry, Gazzaniga, and others were able to demonstrate a left hemisphere preference for linguistic tasks and a right hemisphere preference for visual-spatial tasks.

Problems may arise in interpretation of the findings. Commissurotomy, or split-brain, patients involved in these studies were also epileptics, and it is difficult to ascertain what effects the disease 
might have had on cerebral organization. Since the two hemispheres do not function independently in the normal brain, care must be exercised in equating split-brain hemispheric functioning with that of the intact brain (Cohen 1977). Still, the correlation of findings in split-brain and unilateral-brain damage studies is too close to ignore. The efficiency of each hemisphere in a normal brain can be tested by channeling the stimuli primarily to one or the other hemisphere through the opposite visual or auditory pathway. The underlying assumption in such studies is that the hemisphere which is best suited to a particular task will respond to the information more quickly than will the less adept hemisphere. These studies (Cohen 1977), Springer and Deutsch 1981, Gazzaniga and LeDoux 1978), although not without methodological difficulties, complement split-brain and unilateral-brain damage studies, bolstering the case for two hemispheres which are similar in structure, yet dissimilar in function.

Each hemisphere has a specialized cognitive style; the left hemisphere utilizes a logical mode for which words are an excellent tool, and the right hemisphere uses a holistic, gestalt mode which is suited for spatial relations (Brooks 1978).

Robert Sylwester has dubbed the left hemisphere the "rational mind" because it "analyzes input sequentially, abstracts relevant details, and associates verbal symbols to arrive at a solution" (Sylwester 1978). He describes the right hemisphere as the "metaphoric mind." It processes all data simultaneously without going through logical steps. It seeks metaphors or analogies out of previous experiences. The left hemisphere has been described as the analytic, deductive, sequential, specific, verbal processor (Wittrock 1977). Many of the key words pertaining to left hemispheric processes are similarly used to 
describe an articulated perceptual style in which an individual perceives specific details as discrete from the organized field (Witkin 1967); field-independence, which is characterized by attention to details within the overall framework; and an analytic cognitive style, which abstracts details from the whole and organizes them sequentially. The right brain has been described as the inductive, synthetic, simultaneous, visual processor (Wittrock 1977). These adjectives have also been applied to a field-dependent cognitive style which is characterized by sensitivity to the "big picture" rather than its component parts, and by a need for external direction and organization. These characteristic features of right-brain processing are similarly those ascribed to synthetic or holistic cognitive styles, which are defined in the literature by a lack of logical progression (Entwhistle 1979), a limited sense of psychological differentiation, and a gestalt ability to bridge gaps and fill in missing parts (Galin 1976).

For the purposes of this study, the term "left-brain" cognitive style will be used as a label for the type of information-processing strategies associated with the left hemisphere. Similarly, the label "right-brain" cognitive style will refer to strategies associated with right hemispheric functioning. Table I summarizes the aspects of right and left cognitive styles.

It is important to note that the distinction between hemispheres is not based upon content but upon process variables. Laterality, or the tendency of one hemisphere to dominate information processing, is not task-specific. Rather, the hemisphere which is activated in any given situation is contingent upon the individual and the situation. Both sides are capable of processing the same information; if this were 
not true, how could one explain the fact that the right hemisphere can be reprogrammed to sustain linguistic development following extensive early damage to the left hemisphere (Gazzaniga and LeDoux 1978)?

TABLE I

CHARACTERISTICS OF LEFT AND RIGHT

HEMISPHERIC FUNCTIONING

\section{Left}

Governs language production, speech, writing

Sequential, analytical, time-ordered, serial

Relies on facts and relationship between them

Inductive

Analyzes information for detail

Developed differentiation
Right

Visual-spatial activities

simultaneous, holistic, synthetic thinking

Organization of whole dominates perception of parts

Deductive

Analyzes for overall picture

Governs perception of meaning

To term the left hemisphere the verbal processor and the right hemisphere the picture processor is a vast oversimplification (Lutz 1980). Both hemispheres work in synchrony to process information, but differ as to the approach they use. The left hemisphere analyzes input for specific detail, while the right hemisphere seeks out general characteristics and the total organization of the parts.

The nature of the required response is frequently the catalyst which determines which hemisphere will be called into action. In an 
experiment described by Springer and Deutsch (1981), split-brain patients were asked to match blocks with opened-up, two-dimensional representations. An error analysis showed that the right hand (left hemisphere) was more adept at matching patterns which could be described verbally, while the left hand (right hemisphere) made matches on the basis of visual properties not easily described verbally. This experiment is an example of how each hemisphere is programmed to process material in a particular manner. The critical factor seems to be the nature of the required response rather than the nature of the incoming information.

To summarize, cognitive style might be defined as the tendency of an individual to use a particular hemisphere in perceiving and organizing his environment. This combines the research on lateralization with the classic descriptions of perceptual styles (field dependence/field-independence and cognitive styles (global/articulated, synthetic/analytic, serialistic/holistic. An individual with a left-brain cognitive style would tend to be more field-independent, to analyze information for general characteristics and to process it simultaneously without a logical step-by step progression.

This is consistent with personal observations in both the migrant and regular classroom. Pablo, in substituting "Funny John" for "Silly Sam" did indeed seem to be attending to the meaning of a word rather than its phonetic components. The next step was to examine the literature on cognitive style to see if there was any evidence supporting these observations that Mexican-American and Anglo-American children process information differently.

Several cross-cultural investigations have been conducted comparing 
the perceptual style of Hispanics to that of non-Hispanics. Witkin and Berry (1975) found Mexican-American children performed in a more fielddependent manner than Anglo-American children. In a study measuring the relationship of field dependence to reading and math achievement, AngloAmerican children were found to take a more analytic approach to stimuli than Mexican-American children, who favored a more global approach (Buriel 1978). Results of a study of the cognitive styles of three subcultural groups in Houston (Ramirez and Price-Williams 1974) again showed a higher degree of field dependence in blacks and Mexican-Americans. This tendency toward field dependence seemed to decrease with acculturation (Castaneda, Ramirez, and Herold 1975).

It is important to note, however, that "every human brain is capable of more than one kind of logical process but (that) cultures differ with respect to processes used to deal with various situations" (Springer and Deutsch 1981). This is the nucleus around which this thesis will revolve. 


\section{CHAPTER III}

THE EFFECTS OF LANGUAGE ON COGNITIVE STYLE

Language is one factor cited as having a major effect on cognition. Language provides the labels (vocabulary) for the thing one wishes to talk about and the rules (grammar) for combining lexical units. That the world is perceived and experienced differently by individuals of different linguistic backgrounds gives rise to the theory that language is the source of perceptual differences.

The discussion of the relationship between language and cognitive style will revolve around two central aspects of language: structure and usage. Structure refers to the actual linguistic forms, vocabulary and syntax, which comprise language. Usage, on the other hand, refers to the tendency of an individual to use certain of these forms in communicating his or her thoughts. More simply put, structure consists of those words and grammatical forms available to all speakers of a particular language. The words and grammatical structures which an individual chooses to use in the act of communication constitute usage.

\section{STRUCTURE}

Theories as to how linguistic structure affects cognition range from the relatively conservative position that language shapes neurological pathways by nurturing, or failing to nurture, hemispheric differentiation to the more extreme position that language actually structures reality. 
As stated earlier, the left hemisphere is the center which controls orderly, rational thought. "Language is the first linearity in a world controlled by logic, sequence, and interdependent structure" (Samples 1976). The Western emphasis on logic and structure may nurture the differentiation of the two cerebral hemispheres. If this is true, one would expect to find less differentiation of cerebral functions in cultures without a dominant compulsion toward linear, rational thought (Samples 1976).

This assumption is supported by the work of Tsunoda on brain function in East and West Cultures (Sibatani 1980). Working with speech damaged patients, Tsunoda devised a series of dichotic listening tests designed to function independently of the subject's conscious awareness. Tones triggered by a Morse-code tapping process were fed back directly to one ear while a slightly delayed signal was fed to the other. In addition to pure tones, words, animal noises, and Japanese and Western musical instruments were fed into each ear. The loudness of the signal was increased until it interfered with the subject's ability to continue the tapping process. The purpose was to "ascertain whether or not one hemisphere predominated in registering this interference "(Sibatani). One of Tsunoda's discoveries was that the Japanese brain and the Western brain divide up the labor of processing sensory input in widely different ways. He found that the Japanese process far more sounds in a single hemisphere than do Westerners, placing a tremendous burden on the verbal hemisphere.

There is reason to be cautious about unconditionally accepting all of Tsunoda's generalizations. His treatise The Japanese Brain: Brain Function and East-West Culture has not yet been translated into English, 
so its contents must be examined on the basis of secondary sources. However, if his theories are correct, the possible implication for sociobiology will have to be considered: the neuropsychological patterns of an individual's brain and the resulting cognitive orientation may be influenced by the structure of the language which he or she learns.

\section{LINGUISTIC DETERMINISM}

At the other end of the spectrum is the theory of linguistic determinism, as embodied in the work of Benjamin Whorf. Whorf begins with the observation that individuals from different linguistic backgrounds perceive reality differently, and then proceeds to the generalization that language is the cause of these perceptual differences (Cole and Scribner 1974). Whorf contends that one's impressions of reality are organized according to one's linguistic structures and that these structures are related to attitudes toward time, quantity, and other cultural variables. For example, the English language divides reality into "objects (nouns) which undergo or perform actions (verbs)", thus isolating the individual from his surroundings (Benderly 1981). Whorf contrasts the structure of English with that of Hopi which perceives experience as a "flow of eventuations of varying lengths" (Benderly). Unlike English, Hopi structure tends to immerse the individual in the environment, thus fostering a more holistic or right hemisphere cognitive style. A study conducted with persons of varied backgrounds and abilities showed that Hopis did indeed rely more heavily on right-brain processing than did Anglo subjects (Springer and Deutsch 1981). However Blacks were also 
found to favor right brain processing, suggesting that language structure may not be the sole factor responsible for cognitive differences. Whorf's theory of linguistic determinism equates the presence or absence of a lexical distinction with the presence or absence of a corresponding perceptual distinction (Cole and Scribner 1974). "The ease with which a distinction is expressed in a language is related to the frequency with which its referent perceptual distinction is required in everyday life" (Cole and Scribner). The more than twenty Eskimo terms for snow are frequently cited as illustrative of this principle.

There are obvious flaws in this sort of logic. Eskimos need to make more judgments about snow; therefore, they have more words for it. People from temperate climates do not need to make as many decisions about snow, but this does not ipso facto mean that non-Eskimos are unable to distinguish between the different kinds. The fact that the Eskimo terms can be translated into English refutes this assumption (Cole and Scribner).

Since language is used as an instrument for assessing cognitive processes, it is easy to equate linguistic distinctions with perceptual distinctions, although such facile reasoning can lead to inaccurate conclusions. Visitors to the Pacific Northwest are frequently unfamiliar with the nomenclature for pears, yet one should not assume that because they cannot make the verbal distinction they are similarly incapable of making the visual distinction between the long-necked russet Bosc, the round green d'Anjou, and the classic shaped yellow Bartlett. While the presence of a lexical term may facilitate the expression of perceptual distinctions, it does not serve as proof their existence.

Tentative support of linguistic determinism could be drawn from the results of the Castaneda study (1975), which showed that cognitive style 
changes with acculturation. Mexican-American children from families with a more traditional orientation showed greater field-dependence than those from atraditional homes. As the families became 'mainstreamed' into the Anglo culture, the dominant language shifted from Spanish to English and the degree of field-independence increased proportionately. However, caution must be exercised in interpreting the results of such studies, since Spanish and English are linguistically similar. Both descend from the same Indo-European rootstock, both distinguish between noun functions and verb functions, and both favor the western predisposition toward linear thought. One would thus expect to find similar patterns of cerebral organization in both Spanish and English speakers. The linguistic similarities between the two languages suggest that factors other than language structure may be responsible.

Those who favor structure as a major determinant of cognitive style argue that cognitive processes can be only as complex as the linguistic structures employed to express them. However, advocates of the structural school of thought fail to satisfactorily account for man's ability to translate and borrow terms from other languages and cultures. Nor do they account for the fact that subcultures of similar linguistic origin may have radically different cognitive styles. Since language structure alone fails to adequately account for cognitive differences, other factors must be considered.

USAGE

The second dimension of language to be explored is usage. Anthropologist Basil Bernstein describes two types of communication codes which 
are 'culture-free' or common to all cultures regardless of linguistic background. An elaborated code encompasses a wide range of syntactic options, permitting the expression of a more complex train of thought. Because it has so many alternatives, its structural predictability is low. The meaning is specific to the speaker and forces him to "focus upon another person as an experience different from his own" (Bernstein 1974). In contrast, a restricted code is stereotyped, limited, condensed. The speaker draws from a limited range of syntactic alternatives. The meaning, in order to be understood, depends upon shared experiences. To understand how a restrictive code operates, imagine a bridge game and the 'conversation'between the declarer and his partner. The unstated message is in parentheses.

"One heart." (I have 13 to 17 points and at least five of my cards are hearts.)

"Two diamonds." (I don't have enough hearts to help you, but I do have some diamonds and at least seven points in my hand.)

"Two spades." (Since you don't have many hearts and I don't have many diamonds, what do you have in spades?)

"Three spades." (I have a few good spades, so maybe we can work something out.)

"Four spades." (If we can get ten tricks, we'll get 100 points, so let's go for it.)

"Pass." (Okay, let's!)

The lexical as well as the syntactic alternatives are strictly limited in bridge; still, it illustrates how a restricted code operates. The message is readily understood by the partners, but would be complete1y unintelligible to a non-bridge player.

A restricted code is available to all members of a society, because 
the conditions which generate it are universal (Bernstein 1974). Like all language it operates as a form of social relation where individuals share common interests. However, the extent to which experiences are shared and expectations clearly defined and understood determines the degree of verbalization necessary to communicate a thought. If there is a great degree of overlap in experiences, the need for verbal elaboration is reduced; this condensed form of communication is what Bernstein refers to as a restricted code.

While all individuals have access to a restricted code, the same does not hold true for an elaborated code; the lack of an elaborated code may inhibit the development of a verbal-analytic cognitive style.

If language usage is a major determinant of cognitive style, and if the conditions which generate a restricted code are culture-free, then why do some subcultures favor the use of a restricted code while others utilize an elaborated code? To answer this question, one must examine non-linguistic factors which play a role in determining the type of code used by a given person.

Language is the end-product of the socialization process (Hess and Shipman 1965). The structure of the social system in which one lives influences the type of code which he or she will bring to the communication process. Different social structures foster the development of different linguistic codes. Through speaking and listening, the child learns the requirements of the social system in which he or she lives. (Bernstein 1974). In turn, his intellectual and affective orientation is determined. The stimulation provided in a child's early encounters with his or her environment sets the pattern for later behavior.

If language is the result of the socialization process and if 
cognitive style is determined by the use of a particular linguistic code generated by that process, then one must examine the socialization practices of Mexican-Americans and Anglo-Americans to learn why individuals from those subcultures have different linguistic codes. This will be the focal point of Chapter IV. 


\section{THE RELATIONSHIP BETWEEN SOCIALIZATION PRACTICES AND LINGUISTIC CODE}

It is important to note that the two linguistic codes described in Chapter III result from social relationships and not from intelligence quotient (Bernstein 1974). Cultures do not appear to differ with respect to the cognitive processes themselves (Springer and Deutsch 1981). Every human brain is capable of more than one sort of thought. It is culture which provides the motivation for one's behavior and hence, for the cognitive style preferred by that person (Wiseman 1980).

An infant's brain is characterized by plasticity. One's early encounters with the environment affect the development of cognitive processes and the preference for a particular cognitive style. Adeptness with a particular modality is developed through experience. Earlier it was stated that cognitive style and modality preference are not equivocal. However, modality preference may serve as an indicator of cognitive style. A child who is particularly adept at visuospatial activities, for example, may be demonstrating a preference for a right hemisphere or global cognitive style, since it is the right hemisphere which controls visuospatial processing.

Perceptual styles depend upon environmental stimulation for proper development (Restak 1979). If one or the other hemisphere is not adequately stimulated, its potential for development may be hampered. Different social environments "stimulate, reinforce, and make functional" 
the development of a particular cognitive style while inhibiting or restricting the development of another (Cohen 1969).

Hess and Shipman (1965) describe two types of family control, position or status-oriented and person-oriented, which may influence cognitive style. In a status-oriented environment behavior is regulated in terms of role expectations with little regard for individual personality traits. One is expected to conform to the requirements of one's status. A child may behave in a particular manner because "boys do this" or because "that's what girls are supposed to do." Behavior is controlled by imperatives, and there is little opportunity for decision-making by the child beyond the boundaries of compliance/non-compliance.

In a person-oriented environment, allowances are made for the uniqueness of a person, and that uniqueness modifies the demands of status. Behavior is justified in terms of feelings, abilities, and the individual situation. A child from a person-oriented home is given more opportunity for decision-making, which in turn favors the development of an elaborated code.

The amount of verbal interaction varies in status and personoriented environments. Where behavior is regulated by role expectations and that role is clearly defined and mutually understood, there is little need for verbal elaboration. Such environments foster the development of a restricted linguistic code because the message is defined by, and understood on the basis of, shared interests and expectations. Behavior is not 'mediated by verbal cues which offer opportunities for using language as a tool" (Hertzig 1971). Elaborated codes are more likely to be found in person-oriented environments where, because one takes into consideration such variables as feelings and personality traits, 
there is a greater need for qualitative verbalization.

Language usage, then, as reflected by elaborated and restricted communication codes, is one of the major factors affecting cognitive style. If, as suggested by Bernstein (1974), linguistic codes are generated by different social structures, one must compare the socialization practices of Mexican-Americans and Anglo-Americans to understand why their perceptual styles appear to differ.

Buriel (1978) has shown a greater tendency toward field-dependence among Mexican-Americans and a correspondingly higher rate of field independence among Anglo-Americans. Field-dependence/field-independence is an aspect of cognitive style characterized by the degree of ease with which one is able to perceive himself as separate from his environment or a stimulus as discrete from the surrounding field. It should be noted that field-dependence and field-independence represent opposite poles on a spectrum and that most individuals fall somewhere inbetween these two extremes. The extent to which a person gravitates toward one or the other end of this continuum is a measure of his field-dependence or field-independence. Among factors cited in the literature as influencing this psychological differentiation are the opportunity for separation or independence given the child by his family, the ways in which adults treat the child's impulses (Cole and Scribner 1974), the stress placed upon conformity and obedience (Witkin and Berry 1975), and the degree of emphasis on cooperation and group affiliation (Ramirez and Price-Williams 1974).

As stated earlier, field-dependence stems from environments where there is a heavy emphasis on respect for, and obedience to, authority (Witkin and Berry 1975). Characteristics of field-dependence described 
by Witkin and Berry are a greater tendency to submit to authority, greater attention to social cues, a limited sense of separate identity, and a greater need for external direction (Blanton and Bullock 1973, Witkin and Berry 1975). Field-independence is characterized by such left hemisphere traits as a highly-developed sense of differentiation, attention to specific characteristics or details of an object, and a tendency to impose structure upon a field when little or no organization is inherently present. One would be most likely to encounter fieldindependence in homes where children are encouraged to establish their own standards of behavior and to participate in decision-making (Hess and Shipman 1965). These are the person-oriented homes in which one would also find a tendency to rely upon an elaborated rather than a restricted communication code.

A study of three ethnic groups in Houston (Ramirez and PriceWilliams 1974) revealed a greater degree of field-independence in subcultures characterized by formally-organized family and friendship groups, and a greater degree of field-dependence in the'shared-function' groups which emphasize family and group affiliation. Parents of the subjects participating in the study were asked to fill out questionnaires detailing religious affiliation, childrearing practices, socioeconomic level, and family organization patterns; these questionnaires formed the basis for deciding whether an individual had more in common with formal or with shared-function groups. Of the three groups studied, Anglos had more in common with formal structure groups (and scored in a more fieldindependent direction, while Blacks and Mexican-Americans identified more with shared-function groups and showed a greater degree of fielddependence. 
Within the Mexican-American community, the degree of field-dependence/field-independence parallels the degree of acculturation into the Anglo-American society (Castaneda, Ramirez, Herold 1975). Children from traditional homes were found to perform in a more field-dependent direction than their peers from dualistic and atraditional homes. A traditional home is defined as one in which the primary language is spanish and which stresses interpersonal relations and orientation toward family or group needs. An atraditional home is one in which the language, customs, value system, and family structure more closely resemble those of Anglo society. Dualistic homes are those which are 'in transition' and exhibit characteristics of both the traditional and atraditional homes. As an individual shifts from one type of social organization to another, there is a corresponding shift in language and cognitive styles. Reference has been made to Anglo-American and Mexican-American value systems and patterns of social organization. Table II summarizes some salient characteristics of Mexican-Americans and Anglo-Americans commonly described in the literature.

The characteristics described as being 'typically' Anglo seem to reflect left hemisphere cognitive traits, while the 'typical' MexicanAmerican traits are characteristic of right hemispheric processes. The left hemisphere is time ordered and sequentially-oriented; Anglo-Americans tend to place more emphasis on time schedules than do Mexican Americans. The left hemisphere processes information for individual details; Anglo-Americans are characterized as valuing individuality. The left hemisphere is the verbal-processor; the Anglo-American tendency to encourage questioning fosters verbal behavior. 
FEATURES OF MEXICAN-AMERICAN AND ANGLO-AMERICAN VALUE SYSTEMS

\section{Anglo-American}

Emphasis on individuality

Person-oriented family control

Formally-organized family and friendship groups

Encouraged to question values

Adherence to time schedules

(De Blassie 1976)

\section{Mexican-American}

Emphasis on cooperation

Status-oriented family control

Informally-organized family and

friendship groups

Respect for authority

Nonadherence to time schedules

At the other end of the spectrum, the Mexican-American tendency to emphasize the group rather than the individual corresponds to the right hemisphere's focus on the "big picture" rather than on individual details. Mexican-Americans do not appear to order their lives according to rigid time schedules; neither does the right hemisphere order information in the sequential, time-ordered manner of the left. Again, these characteristics represent extremes on a continuum. In reality, most individuals fall somewhere between opposing poles.

However, the characteristics described in Table II may not be crosscultural variables but functions of socio-economic class. Mexicans who emigrate to the United States may not be at all representative of the larger Mexican population in terms of value systems, linguistic code, or cognitive style. The fact that an analytic cognitive style tends to dominate among middle class individuals and that a relational or synthetic 
style is more prevalent among low-income families supports this premise (Cohen 1969, Lesser 1971).

Several investigations have found that subcultures within the United States are characterized by a predominant cognitive mode; the middle class are likely to use a verbal-analytic mode...the urban poor are more likely to use a spatial-holistic mode (Galin 1976).

These studies suggest that there is a correlation between socioeconomic status and socialization practices. The relationship between economic background, social organization, and liguistic code will be examined more closely in Chapter $\mathrm{V}$. 
CHAPTER V

\section{ACCULTURATION, LINGUISTIC CODE, AND COGNITIVE STYLE}

In Chapter III two linguistic codes, elaborated and restricted, were described which influence the development of cognitive style. It was noted that all human beings have access to a restricted code by virtue of the universal social conditions which generate it (Bernstein 1974). However, it may be that "a considerable section of our society has access only to this (restricted) code by virtue of class background" (Bernstein 1974). This chapter will explore how class background or socioeconomic class may affect one's communication code and, subsequently, one's cognitive style.

Economic conditions place demands upon a family, demands which are reflected in its patterns of social organization. Where wages are low and housing costs high, one may encounter informally-organized extended families. In visits to Mexican-American homes, the investigator encountered a wide range of living arrangements. In many homes older married siblings remained under the parental roof because separate housing was either unavailable or unaffordable. In others, cousins, nieces, and nephews with no means of support comprised part of the extended-family, as did elderly aunts, uncles and grandparents.

In such large loosely-structured groups, child care is generally delegated to whomever happens to be present: a parent, grandparent, cousin, or older sibling. Low-income families are frequently, by necessity, 
shared-function families in which everyone contributes to the upkeep of the group. A 15-year-old skips school to help his father pick fruit; an 8-year-old accompanies his mother to the packing house at night; a 9-year-old is left to care for younger siblings while the parents work; children who are no longer living at home may be expected to contribute part of their earnings to their parents.

Cooperation is vital to the functioning of loosely-organized family units. Behavior is more likely to be defined in terms of role expectations rather than in terms of individual personalities. Respect for authority is considered a greater good than individual achievement among the families visited by this investigator. Survival is paramount; education and other goals are often of secondary importance.

Where people share the same goals and expectations there is a reduced need for verbalization (Bernstein 1974). Roles are strictly defined and behavior is regulated by imperatives, allowing very little opportunity for decision-making or verbal interaction by the child (Hess and Shipman 1965). Children are expected to conform and to obey. Learning is done through observation rather than by explanation. The child in such an environment is not encouraged to perceive himself as separate from others; he is regarded as an integral part of the whole. All these factors may favor the development of a restricted commuication code and a less-developed sense of differentiation.

A child's early encounters with his cognitive environment may have a great impact on the course of his mental growth (Hertzig 1971). In its early stages of development, the human brain is very malleable (Gazzaniga and LeDoux 1978). Modality and cognitive adeptness is developed through experience. If auditory-verbal performance is encouraged, development 
of a left hemisphere cognitive style is greatly enhanced. Verbal explanations, use of inquiry, and use of language as a tool for labelling and ordering stimuli in the environment all contribute to the selective reinforcement of an analytic, differentiating cognitive style (Wiseman 1980). Conversely, if auditory-verbal performance is not stressed during a child's early development, then he will be more likely to attend to visual clues, selectively reinforcing a right hemisphere or synthetic cognitive style. However, while the right hemisphere "appears to develop similar levels of ability in radically different cultural groups. ..development of the left hemisphere is depressed by lack of educational opportunity" (Springer and Deutsch 1981).

In summary, demands placed upon an environment by economic class may affect the development of one's linguistic code. Restricted codes are frequently the norm in shared-function households where the demands of the group overshadow the needs of the individual. A restricted code reduces the need for verbalization, at the same time contributing to reinforcement of the visual modality which is most frequently associated with a right hemisphere cognitive style. Thus, a global style of conceptual organization is favored in homes where, by virtue of socioeconomic class, there is little need or opportunity for verbal interaction.

\section{SOCIOECONOMIC LEVEL}

As a family makes the transition from a traditional orientation to an atraditional one, many changes occur which may account for the accompanying shift in cognitive style. One of the major changes which often coincides with the mainstreaming of a family into the Anglo culture is a 
change in economic level. Financial instability is a hallmark of homes of the 'active' migrants who have lived in the community less than one year. Housing is generally either a small cabin in a camp, or with relatives. Wages are minimum and employment is sporadic. These conditions generate the type of shared-function grouping described earlier in the chapter. Families live, eat, sleep, and work together in close proximity to one another. Life in such close quarters dramatically reduces the need for verbal interaction. A distinction must be made here between talk and communication. Verbal interaction, as it is used here, refers to the process of sharing ideas and communicating thoughts. It should not be confused with the steady flow of words found in many households. Quantity of verbalization is not necessarily synonymous with quality. If economic conditions in a particular area are bleak, the migrant family usually continues to travel from area to area, living in sharedfunction groupings. If the outlook is promising, they may choose instead to remain in one place and seek steady employment. Wages increase with experience and seniority; increased wages enable a family to obtain less crowded living quarters with more opportunity for privacy. The need for shared-functioning decreases with an improved standard of living.

It is difficult to determine if shared-function groupings are dictated by economic condition or encouraged by cultural values. Familial respect is a highly-valued goal among Mexican-American families (Ramirez and Price-williams 1974), but it may be that the goal has evolved from economic necessity. One cannot absolutely equate socioeconomic factors with loosely-organized family groupings, although it is often the case that the two go hand-in-hand. Further studies are needed to determine if it is the value system or economic necessity which gives rise to co- 
operation and shared function groupings.

\section{BILINGUALISM}

Another change which accompanies the shift from traditional orientation to atraditional is bilingualism. Bilingualism may effect changes in cognitive style by actually altering patterns of cerebral organization and forcing an individual to develop and adopt an elaborated code. (Benderly 1981)..

As stated earlier in the paper, in the Western brain the left hemisphere appears to be the center which controls language. Learning a second language imposes an additional burden on the cortex space of the left hemisphere. The brain of a bilingual person must develop a system for keeping the two systems separate and for retrieving the correct word from duplicate sets. Consequently, he or she may be forced to attend more closely to various input cues. This emphasis on linguistic stimuli may enhance a child's verbal abilities, as well as encourage hemispheric differentiation.

In many dualistic and atraditional homes, the child's bilingualism poses new communication problems, particularly if the parent maintains only limited fluency in the second language. This investigator has visited over a dozen homes where the children speak a curious mixture of the two languages, freely substituting words from one when they are unable to think of the counterpart in the other, or when the counterpart could not be translated. The restricted code of the traditionallyoriented family is often no longer adequate for communication. There is a need for increased verbal interaction in order to relay a message. These conditions generate a need for an elaborated code if communication 
is to succeed.

\section{EDUCATION}

Acculturation imposes changes in cognitive style through the influence of second language acquisition and changes in economic level. A final factor which should be considered in light of its impact on cognitive style is education.

Education can affect cognitive processes in diverse ways. Many researchers feel that American public schools favor a left hemisphere or field-independent approach to instruction (Ornstein 1972, Cohen 1969, Ramirez 1973) because"if our society is predominantly left hemisphere, then so too should our education system, and there is overwhelming support that it is" (Brooks 1978). This left hemisphere preference is reflected, according to the literature, in a sequential, part-to-whole instructional format and in an emphasis on analytical thinking. According to Ornstein (1972) intellectual training is oriented toward the analytic with the result that one tends to perceive the fragments instead of the whole. "The scientific and technical world view of our time contains a strong bias toward the rational thought processes characteristic of the left hemisphere" (Fischer and Rhead 1974).

As stated earlier, perceptual styles are dependent upon stimulation for their development (Restak 1979). The use of language as a tool for labelling and manipulating stimuli enhances the tendency of the left hemisphere to dominate information processing, while a lack of verbal stimulation of the educational environment to have an effect on one's cognitive style, and there is evidence that it does (Cole and Scribner 1974). The development of new intellectual tools such as perceptual analysis can 
be the direct result of education (Cole and Scribner).

Changes in linguistic code induced by social organizational

patterns, second language learning, and education may account for the shift toward field-independence documented by Castaneda, Ramirez, and Herold 1975). All three factors trigger changes in communication code and consequently in cognitive style, through increased stimulation of the left hemisphere. There appears to be a significant correlation between verbal stimulation and conceptual organization.

Restricted communication codes appear to be more prevalent among lower income families where, because of economic conditions, living quarters are closely shared and roles strictly defined. Under such conditions, the need for elaborated verbal interaction is reduced, resulting in decreased left hemisphere stimulation and the less-developed sense of psychological differentiation characteristic of field-dependence. Changes in economic level, when accompanied by changes in living conditions, directly affect linguistic code by increasing the need for verbal interaction.

Second language learning and education similarly affect linguistic code and cognitive style through increased stimulation of the verballyoriented left hemisphere. If language is used as a vehicle for processing one's experiences, the left hemisphere will be properly stimulated and one will be able to retain a potential for left-hemisphere thought. This potential cannot be developed without adequate verbal stimulation.

Language usage, then, appears to be a key factor affecting cognitive development; it, in turn, may be influenced by social organizational patterns resulting from economic level. 
CHAPTER VI

CONCLUSION AND RECOMMENDATIONS

The purpose of this thesis is threefold:

1) to identify learning styles as observed by the investigator

2) to define cognitive style in terms of recent neuropsychological research

3) to identify major factors which affect cognitive style.

Observations made by the investigator that Mexican-American children tend to utilize different information-processing strategies were confirmed by results of studies which show Mexican-Americans to perform in a more field-dependent direction than Anglos. Field-dependence and field-independence are a dimension of cognitive style characterized by the degree to which one is able to view a stimulus as separate from the surrounding field. To the extent that one is able to do this, he is considered to be field-independent. An individual who has difficulty perceiving the parts as differentiated from the overall organization is considered to be field-dependent. Early literature on cognitive style focused on this dimension of perceptual organization.

Recent neuropsychological research has shed new light on cognitive processes, and it now appears that field-dependence/field-independence is but a single facet of cognitive style. Cognitive style itself is more than just psychological differentiation; it is a function of hemispheric differentiation which results from the interaction of an individual with his cognitive environment. 
At birth the human brain is incompletely developed. It maintains a potential for specialization of hemispheric function, but is dependent upon environmental stimulation for development. In most right-handed, Indo-European speakers language is centered in the left hemisphere, while the right hemisphere is the site for visuospatial activities. Because language is logical, partitioned, and serialistic, it follows that the left hemisphere, by virtue of its linguistic capabilities, will be the center for processing orderly, rational, analytical thought. If emphasis is given to linguistic expedients, the left hemisphere will tend to dominate perception and a person will be more adept at part-to-whole learning. The visuospatially-oriented right hemisphere, on the other hand, perceives the organization of the whole rather than the component parts. If the left hemisphere is not sufficiently stimulated, the right hemisphere will dominate information-processing. It is important to bear in mind that although a person may favor a particular processing style, this dominance need not be absolute.

Language is a key factor affecting cognitive style, but theories differ as to whether structure or usage is the determinant. Studies comparing Eastern and Western cerebral structure suggest that the language we learn may actually alter the organization of the brain and the resulting cognitive orientation. The major drawback of this hypothesis is that it fails to account for cognitive differences among speakers of the same language. It is further contradicted by evidence showing a correlation between acculturation and changes in cognitive style. It appears that language usage, and not structure, may be the more significant factor affecting one's cognitive orientation.

Language usage, and subsequently, cognitive style, can be influ- 
enced by both cultural and socioeconomic variables. Cohen (1969) posits that factors within the social framework "stimulate, reinforce, and make functional the development of one style of conceptual organization and constrain and inhibit others." She maintains that, as a result of social organizational factors, an analytic cognitive style is less prevalent among low-income families. Lesser (1971) similarly found that the middle class are more likely to favor a verbal-analytic style while individuals from low-income environments tend to use spatialholistic processing strategies. This dichotomy may be partially explained by the observation that:

the right hemisphere appears to develop similar levels of ability in radically different cultural groups while the left hemisphere is depressed by lack of educational opportunity. (Springer and Deutsch 1981).

Hertzig (1971) describes the cognitive environment of the disadvantaged child as one in which language is not used as a tool for labelling or processing experiences.

These studies suggest a strong correlation between low-income homes, language usage, and cognitive style. If a child is sufficiently stimulated by deep-structure verbal interaction, he will retain a potential for a left hemisphere cognitive style. Education, communication and social organizational factors all work in concert to foster or inhibit the development of hemispheric specialization. To the extent that verbal behavior is encouraged, the left hemisphere will tend to dominate information-processing. If it is not sufficiently stimulated the individual may attend more closely to visual and social cues, thus selectively reinforcing a right hemisphere cognitive style.

Hemispheric specialization, then, appears to be a function of 
language usage. There is a direct correlation between the type of communication code used by an individual and his preferred mode of information-processing. To the extent that language usage is encouraged as a vehicle for exploring and explaining phenomena in one's environment, discussing one's experiences, and communicating one's thoughts, an individual will foster an analytic, left-hemisphere cognitive style. If, on the other hand, quality verbal interaction is not stressed, the holistic right hemisphere processing style may, by default, tend to dominate.

Language usage appears to be influenced by social organizational factors which may arise as a result of economic class. Environmental conditions seem to dictate the type of code that an individual will use for communication. If behavior is rigidly regulated by role expectations, as several investigators have suggested may be the case in low-income environments, a more restricted communication code may result, thus inhibiting the tendency toward a left hemisphere cognitive style.

\section{IMPLICATIONS}

One study has shown that Anglo-American perform in a more fieldindependent direction and are academically higher than Mexican-Americans (Buriel 1978). On the surface, this suggests that a left hemisphere cognitive style may be more desirable than a right-hemisphere cognitive style. Cognitive style is not a function of intelligence, however. The superiority of a given style is contingent upon the situation (Garner 1979). Since most individuals possess a cognitive style which lies somewhere along a continuum between left and right, it is the nature of the response which determines the processing strategies that will be called. 
upon in a given task. If both hemispheres are reasonably adept, then the individual will encounter few difficulties responding. If, however, the hemispheres have developed unequally, the less adept hemisphere may be at a great disadvantage when trying to use a cognitive style with which it has not had sufficient experience.

The "superior" academic performance of Anglo-Americans is probably due, not to left-hemisphere cognitive style alone, but to the fact that linguistic means are most often used to assess academic ability, and to the fact that the educational system seems to favor a left-hemisphere cognitive style.

Current educational practices such as an overemphasis on phonetic analysis and recalling specific details stress verbal-analytic thinking. These verbal-analytical tasks are achieved more easily by left hemisphere learners than by individuals who are right-hemisphere dominant. The extent to which a child's preferred cognitive style is consistent with the cognitive style fostered by the school may determine the degree of academic success he will encounter (Harker 1977). This may partially explain the difficulties encountered by right-hemisphere dominant students in a left hemisphere-oriented school environment.

\section{APPLICATION}

Knowledge of cognitive style and hemispheric specialization can facilitate instruction of all students. By recognizing cognitive differences in children from divergent cultural and socioeconomic backgrounds, teachers can meet their academic needs in a more consistent, systematic manner.

Awareness of cognitive processes and the factors which influence 
their development should serve as a springboard for instructional variability. If a child prefers a holistic right-hemisphere approach to information-processing, he should be taught in a manner which allows him to use his right hemisphere and thus experience success. Yet at the same time, he should be exposed to left hemisphere activities in such a way that he can increase the capacity of the less adept hemisphere. Right hemisphere instruction might include the use of picture clues to facilitate vocabulary retention by activating the visual processes of the right hemisphere. Words should be taught in context rather than constructed phonetically from isolated sounds. The right hemisphere can also be stimulated by providing an outlet for creative expression through art and drama. Right hemisphere children might be encouraged to act out what happened in a story rather than recalling specific details about it. Restructuring instructional practices so that learners are exposed to different cognitive styles is the first step to meeting and serving the educational needs of every student. 


\section{A SEIECTED BIBLIOGRAPHY}

Banville, Dr. Thomas. "Brain Hemisphericity: A High Voltage Topic." Early Years 10 (October 1979): 38 - 41.

Bart, william. Development and Neurological Implications For Relating Cognition and Affect. (Boston: ERIC Document Reproduction Services, ED 190614,1980$)$.

Benderly, Beryl Lief. "The Multilingual Mind." Psychology Today 11 (March 1981): $9-12$.

Bernstein, Basil. "Elaborated and Restricted Codes: Their Social origins and Some Consequences." American Anthropologist 66 (December 1974): $55-69$.

Berry, J.W. "Ecological and Cultural Factors in Spatial Perceptual Development." Canadian Journal of Behavioral Science 3 (4): $324-336$.

Bing, Elizabeth. "Effect of Childrearing Practices on Development of Differential Cognitive Ability." Child Development 34 (September 1963): $631-648$.

Blanton, William E. and Bullock, Terry. "Cognitive Style and Reading Behavior." Reading World 12 (1973): 276 - 287.

Brooks, Rick. Relationship Between Piagetian Cognitive Development and Cerebral Asymetry. (University of Northern Colorado: ERIC Document Reproduction Services, ED 160224 , 1978).

Buriel, Raymond. "Cognitive Styles Amont Three Generations of MexicanAmerican Children." Journal of Cross-Cultural Psychology 6

(December 1975): $417-429$.

Buriel, Raymond. "Relationship of Three Field-Dependent Measures to Reading and Math Achievement of Anglo-American and Mexican-American Children." Journal of Psychology 70 (April 1978): 167 - 174.

Cassell, Russell N. "Split Brain Functioning." Education 99 (Fall 1978): 2 - 7 .

Castaneda, A., Ramirez M., and Herold, P.I. New Approaches to Bilingual, Bicultural Education. The Dissemination and Assessment Center for Bilingual Education, Austin: 1975. 
Cohen, Gillian. The Psychology of Consciousness. London: Academic Press, 1977.

Cohen, R.A. "Conceptual Styles, Culture Conflict, and Non-Verbal Tests of Intelligence." American Anthropologist 71 (February 1969): 828-856.

Cole, Michael and Bruner, J.S. "Cultural Differences and Inferences About Psychological Processes." American Psychologist 26 (October 1971): 867 - 876 .

Cole, Michael and Scribner, Sylvia. Culture and Thought: A Psychological Introduction. New York: Wiley and Sons, 1974.

Cortes, Carlos E. "Chicano Culture, Experience, Learning." In Extracting Learning Styles from Social, Cultural Diversity: A study of Five American Minorities. Edited by Lee Morris. (Norman, Oklahoma: ERIC Document Reproduction Services, ED 158 952, 1978).

Cronbach, L.J. and Snow, R.E. Aptitudes and Instructional Methods: A Handbook for Research on Interactions. New York: Irvington, 1977.

DeBlassie, Richard R. Counseling With Mexican-American Youth. (Austin: Learning Concepts Inc., 1976), $25-48$.

Dixon, Carol N. Matching Reading Instruction to Cognitive style for Mexican-American Children (Santa Barbara, Ca.: ERIC Document Reproduction Services, ED 158 269, 1977).

Dyk, R.B. and witkin, H.A. "Family Experiences Related to Development of Differentiation in Children." Child Development 36 (1965): $21-55$.

Entwhistle, Noel. Motivation, Styles of Learning, and the Academic Environment. (ERIC Document Reproduction Services, ED 190636 , 1979).

Feminello, Francis $x$. Education and Ethnicity: Euro-Ethnics in AngloEthnic Schools. (Chicago: ERIC Document Reproduction Services, ED 183439,1979$)$.

Fischer, R. and Rhead, J. "The Logical and the Intuitive." Main Currents in Modern Thought 31 (1974): $50-54$.

Fishman, J.A. "A Systematization of the Whorfian Hypothesis." Behavorial Science 5 (4) (1960): 323 - 329.

Galin, David. "Educating Both Halves of the Brain." Childhood Education. 53 (October 1976): $17-20$.

Garner, Ruth. The Importance of Cognitive Styles Research for Understanding the Reading Process. (University of Maryland: ERIC Document Reproduction Services, ED 172 148, 1979). 
Gazzaniga, Michael S., and LeDoux, Joseph E. The Integrated Mind. New York: Plenum Press, 1978.

Giordano, Gerard. Neurological Research and Implications for Teaching Bilingual Children to Read (New Mexico: ERIC Document Reproduction Services, ED 144 396, 1977).

Goodman, David A. "Learning from Lobotomy." Human Behavior 7 (January 1978): $44-49$.

Guay, Roland B., and McDaniel, Ernest D. Toward Explaining Sex Differences in Spatial Ability: An Investigation of Selected Cultural and Neuropsychological Factors. (Arlington, Va: ERIC Document Reproduction Services, ED 185 071, 1979).

Harkex, Richard K. Cognitive Style, Environment, and School Achievement: A Cross-Cultural Study (New Zealand: ERIC Document Reproduction Services, ED 161 983, 1977).

Hart, Leslie. "The Three-Brain Concept and the Classroom." Phi Delta Kappan 62 (March 1981): $42-45$.

Hertzig, M.E. Cognitive Studies, Volume 2 (New York: Brunner-Mazel Publications, 1971), 146-148.

Hess, Robert. D. and Shipman, Virginia C. "Early Experiences and the Sociology of Cognitive Modes in Children." Child Development 36 (December 1965): $869-886$.

Hunter, M. "Right-Brained Kids in Left-Brained Schools." Today's Education 65 (November/December 1976): $45-48$.

Iaosa, Luis M. Maternal Teaching Strategies and Field-Dependent/Independent Cognitive Styles in Chicano Families. (Chicago: ERIC Document Reproduction Services, ED 162 762, 1978).

Lesser, Gerald. Psychology and Educational Practice. (New York: Scott, Foresman, 1971), Chapters 10 and 18.

Lutz, Kathryn. Implications of Brain Research for Learning Strategies and Educational Practice. (University of Southern California: ERIC Document Reproduction Services, ED 163 068, 1980).

Ogletree, Earl. J. and Mandajana, Jerry. Cognitive Style of MexicanAmerican Children (Chicago: ERIC Document Reproduction Services, ED 164187,1975$)$.

Ornstein, Robert. The Psychology of Consciousness. San Francisco: W.S. Freeman and Co., 1972 . 
Ornstein, Robert. "The Split and the Whole Brain." Human Nature 1 (May 1978) : $93-99$.

Ramirez, Manuel. "Cognitive Styles and Cultural Democracy in EducatIon." Social Science Quarterly 53 (March 1973) 895 - 904.

Ramirez, M. and Price-Williams, D.R. "Cognitive Styles of Children of Three Ethnic Groups in the United States." Journal of CrossCultural Psychology 5 (June 1974): 212 - 219.

Reed, Rodney Louis. The Relationship Between Language Acquisition and Cognitive Development. (Michigan State University: ERIC Document Reproduction Services: ED 172516 , 1977).

Restak, Richard M. The Brain: The Last Frontier. New York: Doubleday, 1979.

Sagan, Carl. The Dragons of Eden: Speculations on the Evolution of Human Intelligence. New York: Ballantine, 1977.

Samples, Bob. The Metaphoric Mind. (Reading: Addison-Wesley, 1976), $16-26$.

Sibatani, Atuhiro. "The Language We Learn Alters the Physical Operation of Our Brains." Science '80 1 (December 1980): $22-27$.

Sigel, Irving E. and McGillicuddy-Delisi, Ann V. Parental Distancing, Beliefs and Children's Representational Competence Within Family Context (University of Wisconsin: ERIC Document Reproduction Services, ED 194231,1980 ).

Springer, Sally and Deutsch, Georg. Left Brain, Right Brain. San Francisco: W.H. Freeman and Co., 1981.

Stodolsky, Susan S. and Lesser, Gerald. "Learning Patterns in the Disadvantaged." Harvard Educational Review 37 (Fall 1967): 546.

Sylwester, Robert. "Brain Training." Instructor LXXX VIII (October 1978) : $112-116$.

Wiseman, Richard L. Functional systems and Culturally-Determined Cognitive Differences (ERIC Document Reproduction Services, ED $185634,1980)$.

Witkin, H.A. "Cognitive Styles Across Cultures." International Journal of Psychology 2 (1967): $233-250$.

Witkin, H.A., Price-Williams, D., Bertini, M., Christiansen, B., Altman, S.K., Ramirez, M., and Van Mell, J. "Social Conformity and Psychological Differentiation." International Journal of Psychology 9

(1974): $11-29$. 
Witkin, H.A. and Berry, J.W. "Psychological Differentiation in CrossCultural Perspective." Journal of Cross-Cultural Psychology 6 (March 1975): $4-87$,

Witkin, H.A., Moore, C.A., Goodenenough, D.R., and Cox, P.W. "FieldDependent and Field-Independent Cognitive Styles and their Educational Implications." Review of Educational Research 47 (Winter 1971) $1-65$.

Wittrock, M.C. The Human Brain. New Jersey: Prentice Hall, 1977.

Wittrock, M.C. "The Cognitive Movement in Instruction." Educational Researcher 8 (February 1979) 5 - 11 . 